University of Nebraska - Lincoln

DigitalCommons@University of Nebraska - Lincoln

2-13-2008

\title{
Effects of an external electric field on the ferroelectric-paraelectric phase transition in polyvinylidene fluoride-trifluoroethylene copolymer Langmuir-Blodgett films
}

\author{
Matt Poulsen \\ University of Nebraska-Lincoln, map@suiter.com
}

\author{
A. V. Sorokin \\ University of Nebraska - Lincoln \\ Shireen Adenwalla \\ University of Nebraska-Lincoln, sadenwalla1@unl.edu \\ Stephen Ducharme \\ University of Nebraska, sducharme1@unl.edu \\ V. M. Fridkin \\ Institute of Crystallography of the Russian Academy of Sciences, 117333 Moscow, Russia
}

Follow this and additional works at: https://digitalcommons.unl.edu/physicsducharme

Part of the Physics Commons

Poulsen, Matt; Sorokin, A. V.; Adenwalla, Shireen; Ducharme, Stephen; and Fridkin, V. M., "Effects of an external electric field on the ferroelectric-paraelectric phase transition in polyvinylidene fluoridetrifluoroethylene copolymer Langmuir-Blodgett films" (2008). Stephen Ducharme Publications. 40. https://digitalcommons.unl.edu/physicsducharme/40

This Article is brought to you for free and open access by the Research Papers in Physics and Astronomy at DigitalCommons@University of Nebraska - Lincoln. It has been accepted for inclusion in Stephen Ducharme Publications by an authorized administrator of DigitalCommons@University of Nebraska - Lincoln. 


\title{
Effects of an external electric field on the ferroelectric-paraelectric phase transition in polyvinylidene fluoride-trifluoroethylene copolymer Langmuir-Blodgett films
}

\author{
Matt Poulsen, ${ }^{1}$ A. V. Sorokin, ${ }^{1, a)}$ S. Adenwalla,${ }^{1}$ Stephen Ducharme, ${ }^{1, b)}$ and V. M. Fridkin ${ }^{2, c)}$ \\ ${ }^{1}$ Department of Physics and Astronomy and the Nebraska Center for Materials and Nanoscience, \\ University of Nebraska, Lincoln, Nebraska 68588-0111, USA \\ ${ }^{2}$ Institute of Crystallography of the Russian Academy of Sciences, 117333 Moscow, Russia
}

(Received 9 October 2007; accepted 2 December 2007; published online 13 February 2008)

\begin{abstract}
X-ray diffraction and capacitance measurements have been employed to study the structural and dielectric behavior of the ferroelectric-paraelectric phase transition under the influence of a large external electric field. The samples under study are ultrathin $(15-100 \mathrm{~nm})$ Langmuir-Blodgett films of a copolymer of vinylidene fluoride (70\%) with trifluoroethylene (30\%) deposited on aluminum-coated silicon. In situ $\theta-2 \theta$ x-ray diffraction was used to measure the change in interlayer spacing perpendicular to the film surface, corresponding to the (110) direction and indicating that the polymer chains along (001) lie predominantly in the plane, while capacitance measurements were used to monitor the behavior of the dielectric constant of the film. Application of a large electric field, up to $265 \mathrm{MV} / \mathrm{m}$, raises the phase transition temperature and can convert the nonpolar trans-gauche paraelectric phase to the polar all-trans ferroelectric phase in a reversible manner.
\end{abstract} (c) 2008 American Institute of Physics. [DOI: 10.1063/1.2838484]

\section{INTRODUCTION}

Ferroelectricity has been studied actively since the pioneering work of Valasek in the 1920s. ${ }^{1}$ Some of the most interesting characteristics of ferroelectric materials are the spontaneous polarization and consequent polarization hysteresis, as well as the transition to a nonpolar paraelectric phase at a transition temperature $T_{\mathrm{co}}$. Some ferroelectrics are crystalline polymers with polar repeat units, ${ }^{2,3}$ such as the odd nylons $^{4}$ and polyvinylidene fluoride (PVDF) and its copolymers, with, e.g., trifluoroethylene (TrFE) ${ }^{5,6} \mathrm{PVDF}$ and its copolymers have long been studied due to their pyroelectric and piezoelectric properties. ${ }^{7}$ The copolymer P(VDF-TrFE) has the simple linear structure- $\left(\mathrm{CH}_{2}-\mathrm{CF}_{2}\right)_{x}$ $-\left(\mathrm{CF}_{2}-\mathrm{CHF}\right)_{1-x}-$, where the monomers are understood to have a random sequence. ${ }^{8,9}$ The ferroelectric $\beta$ phase of $\mathrm{P}(\mathrm{VDF}-\mathrm{TrFE})$ consists of all-trans chains, ${ }^{2}$ which possess a net electric dipole moment perpendicular to the carbon backbone, directed from the electronegative fluorine toward the electropositive hydrogen. When $\mathrm{P}(\mathrm{VDF}-\mathrm{TrFE})$ is heated through the ferroelectric-paraelectric phase transition, the alltrans chains develop a disordered trans-gauche conformation packed in a centrosymmetric hexagonal structure and a (110) lattice spacing that is about $10 \%$ larger than that of the $\beta$ ferroelectric phase. ${ }^{10-12}$

Application of hydrostatic pressure tends to increase the coercive field ${ }^{13}$ and transition temperature ${ }^{14,15}$ even to the point of converting the transition type from second order to first order in low-VDF content polymers. ${ }^{16}$ It is also possible to convert the paraelectric phase to the ferroelectric phase

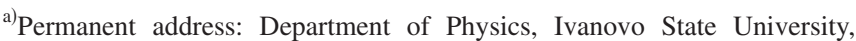
Ivanovo 153025, Russia. Electronic mail: a40in@mail.ru.

${ }^{b}$ Electronic mail: sducharme1@unl.edu.

${ }^{c)}$ Electronic mail: fridkin@ns.crys.ras.ru.
}

through the application of an external electric field. ${ }^{17-19}$ Here we report studies of the combined effects of temperature and electric field on the ferroelectric and paraelectric phases in Langmuir-Blodgett (LB) films ${ }^{20-22}$ of the P(VDF-TrFE) $70 / 30$ copolymer. The relative proportions of the phases were determined from $x$-ray diffraction measurements and correlated with capacitance measurements. Application of a large external electric field converts the films from the paraelectric phase to the ferroelectric phase. This process is reversible and exhibits hysteresis; the film reverts back to the original paraelectric state as the electric field is reduced. This is fundamentally different than previous experiments in which applied electric fields were simply used to irreversibly convert the nonpolar paraelectric phase to the polar ferroelectric phase in multiphased cast films of pure PVDF samples. ${ }^{17}$ Capacitance measurements show that an external electric field increases the ferroelectric-paraelectric phase transition temperature upon both heating and cooling. The conversion between ferroelectric and paraelectric is reversible and is accompanied by a large hysteresis in both temperature and electric field.

\section{SAMPLE PREPARATION}

The Langmuir-Blodgett technique involves the layer-bylayer transfer of $\mathrm{P}(\mathrm{VDF}-\mathrm{TrFE}) 70 / 30$ copolymer from an air-water interface onto a solid substrate such as silicon or glass. ${ }^{23}$ The ultrathin LB films were prepared as follows. The copolymer was thoroughly dissolved to a $0.05 \%$ by weight concentration in acetone and dispersed on a water subphase. The substrates consisted of electronic-grade silicon (111) wafers coated with $50 \mathrm{~nm}$ of aluminum by vacuum evaporation. The Langmuir layer on the water was compressed slowly to a deposition pressure of $5 \mathrm{mN} / \mathrm{m}$ and transferred onto the substrate one layer at a time using the Schaefer horizontal 
(a)
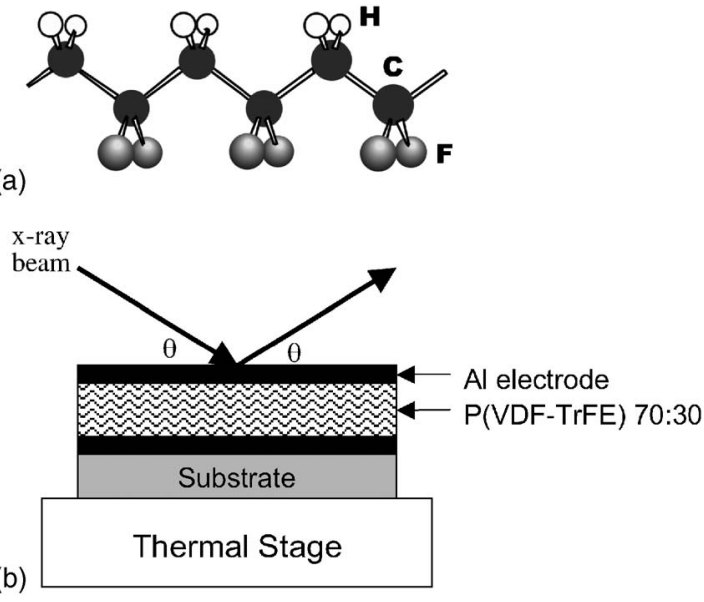

FIG. 1. (a) Structure of the all-trans conformation of the ferroelectric $\beta$ phase. (b) A diagram of experimental setup used in $\theta-2 \theta$ x-ray diffraction measurements.

dipping variation of LB deposition. ${ }^{24}$ In this study, sample thickness ranged from 5 to 150 nominal monolayers (MLs). Upon completion of the LB deposition, an additional $50 \mathrm{~nm}$ of aluminum was then evaporated onto the top of the copolymer film, creating a simple capacitor. Completed samples were annealed in the paraelectric phase at $120^{\circ} \mathrm{C}$ for approximately $1 \mathrm{~h}$ to improve crystallinity, which was confirmed by a significant increase in the (110) x-ray diffraction peak intensity at $25{ }^{\circ} \mathrm{C}$. Additional annealing or field application did not increase the (110) peak intensity further.

\section{EXPERIMENTAL METHODS}

The x-ray diffraction (XRD) and capacitance measurements revealed changes to the crystal structure and dielectric constant, respectively, across the ferroelectric-paraelectric phase transition. Figure 1(b) illustrates the experimental setup used for temperature and electric-field dependent capacitance and XRD measurements. Sample capacitance was measured with a Hewlett Packard 4192A impedance analyzer operating at $1 \mathrm{kHz}$ frequency and $0.1 \mathrm{~V}$ amplitude and recorded as a function of temperature and applied dc voltage. Temperature was monitored and controlled using a platinum resistance thermometer in conjunction with a resistive heating element and a thermoelectric cooler. Upon heating and cooling, the temperature of the copolymer film was swept at a rate of $1{ }^{\circ} \mathrm{C} / \mathrm{min}$ from $(15 \pm 1)$ to $(120 \pm 1){ }^{\circ} \mathrm{C}$. A series of these temperature-sweep measurements was then made with a range of applied voltages.

The XRD measurements were made in the $\theta-2 \theta$ geometry [Fig. 1(b)] with a Rigaku diffractometer with fixed copper anode (wavelength $=1.542 \AA$ ). The (110) x-ray diffraction peaks for the ferroelectric $\beta$ phase $(4.5 \AA)$ and the paraelectric phase $(4.8 \AA)$ are well resolved, making it possible to determine the relative proportions of each phase from the integrated peak intensities. The XRD data were recorded at a series of fixed temperatures ranging from $(30 \pm 5)$ to $(120 \pm 5){ }^{\circ} \mathrm{C}$. Each temperature value was maintained for approximately $1 \mathrm{~h}$ before an XRD measurement was made. As a check at some temperatures, a second XRD scan was made under the same conditions after a few hours to verify that the film structure was stable on this time scale. For the study of field-induced phase conversion at a fixed temperature of $90{ }^{\circ} \mathrm{C}$, the sample was first annealed at $120^{\circ} \mathrm{C}$ for $1 \mathrm{~h}$ to ensure that it was entirely in the paraelectric phase and then slowly cooled to $90{ }^{\circ} \mathrm{C}$.

\section{RESULTS AND DISCUSSION}

\section{A. Phase conversion due to temperature changes}

The ferroelectric-paraelectric phase transition is evident in temperature-dependent capacitance measurements, which exhibit a Curie-Weiss-like maximum in the dielectric constant as the sample is heated or cooled through the phase transition. Figure 2(a) shows the capacitance peaks recorded at zero field from a 50-monolayer P(VDF-TrFE) sample deposited on aluminum-coated silicon. Upon heating, the capacitance of the sample gradually increases as the temperature is increased until a peak in the capacitance occurs at $(100 \pm 5){ }^{\circ} \mathrm{C}$, representing the phase transition from the ferroelectric phase to the paraelectric phase. Conversely, upon cooling a maximum in the capacitance is observed at $(67 \pm 5){ }^{\circ} \mathrm{C}$, indicating a conversion from the paraelectric phase to the ferroelectric phase. The positions and magnitudes of the heating and cooling peaks were insensitive to the rate of heating and cooling and were repeatable on multiple temperature cycles. The capacitance data shown in Fig. 2(a) are in good agreement with previous studies on polymorphous copolymer films ${ }^{6,25}$ and on LB films. ${ }^{19-21,26}$

The structural change across the ferroelectricparaelectric phase transition is clearly evident in the $\mathrm{x}$-ray diffraction data near $2 \theta=19^{\circ}$. Temperature-dependent XRD data recorded with a 50-monolayer copolymer sample are displayed in Figs. 3(a) and 3(b) for the cases of heating and cooling, respectively. One can see that at $30^{\circ} \mathrm{C}$ before heating, there is only the peak at $2 \theta=19.8^{\circ}$, corresponding to a $d$ spacing of approximately $4.5 \AA$. This peak is generally associated with the (110) and (200) reflections in the ferroelectric phase. ${ }^{9,10,12}$ At temperatures well above the heating transition temperature $\left(\geqslant 100^{\circ} \mathrm{C}\right)$, there is only the peak at $2 \theta=18.1^{\circ}$, corresponding to a $d$ spacing of approximately $4.9 \AA$, which is usually associated with the (110) and (200) reflections of the paraelectric phase. ${ }^{9,10,12}$

Phase coexistence and thermal hysteresis are evident in the XRD data as the sample was gradually heated and then cooled through the phase transition. As the temperature of the sample was increased, a shoulder rises on the low-angle side of the $2 \theta=19.8^{\circ}$ ferroelectric peak. The shoulder became a clear and well-resolved second peak at approximately $2 \theta=18.5^{\circ}$ at a temperature of $70^{\circ} \mathrm{C}$. The paraelectric phase peak continued to grow at the expense of the ferroelectric phase peak until the latter disappeared at a temperature of $(100 \pm 5){ }^{\circ} \mathrm{C}$, leaving only the paraelectric phase peak, which has shifted down to $2 \theta=18.1^{\circ}$ as a result of thermal expansion. Conversely, upon cooling from $120^{\circ} \mathrm{C}$, only the paraelectric phase peak was evident down to $(60 \pm 5){ }^{\circ} \mathrm{C}$, where the peak associated with the ferroelectric phase first appeared at $2 \theta=19.6^{\circ}$. Upon further cooling, the paraelectric phase peak diminished and the ferroelectric phase peak grew, but there remained a significant portion of the paraelectric 


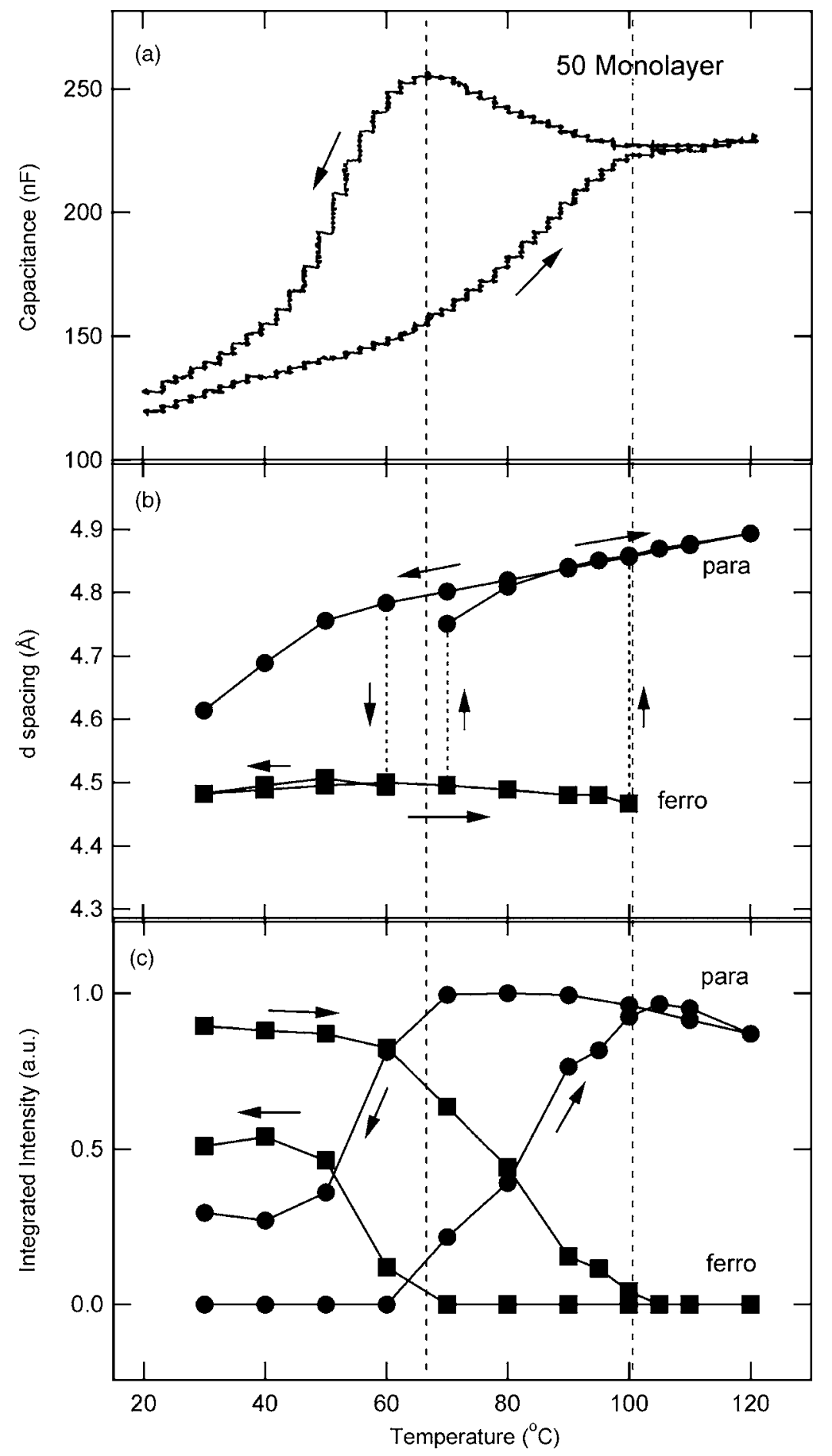

FIG. 2. Temperature dependence of (a) the capacitance, (b) (110) crystal spacing perpendicular to the sample surface, and (c) the integrated intensities of x-ray diffraction peaks from a 50-monolayer LangmuirBlodgett film of P(VDF-TrFE). The circles (-) and squares ( $\square)$ represent the paraelectric and ferroelectric phases, respectively.

phase, still persisting even at a temperature of $(30 \pm 5){ }^{\circ} \mathrm{C}$. The residual paraelectric phase is evident in the rather broad asymmetric XRD peak observed at $30^{\circ} \mathrm{C}$ on cooling [as compared to the data recorded at $30{ }^{\circ} \mathrm{C}$ at the beginning of the heating portion, in Fig. 3(a)]. This gradual conversion between the ferroelectric phase and the paraelectric phase upon heating and between the paraelectric phase and the ferroelectric phase upon cooling is a characteristic feature of phase coexistence. It should also be noted that reducing or increasing the rate of heating or cooling by a factor of 2 had no measurable effect on these results.

The integrated intensities of the Bragg peaks associated with the ferroelectric and paraelectric phases can be used to monitor the relative proportion of the two phases as a function of temperature if it is assumed that the ferroelectric phase and the paraelectric phase have equal structure factors. The XRD data shown in Figs. 3(a) and 3(b) were fitted to a Lorentzian line shape, yielding the position, width, and area of each peak at each temperature. The $d$ spacings and integrated intensities of these peaks are shown in Figs. 2(b) and 2(c), respectively. The temperature dependence of the interlayer spacings, calculated from the XRD peak positions in Figs. 3(a) and 3(b), is shown in Fig. 2(b), where the region of ferroelectric-paraelectric phase coexistence is readily observed. The coexistence region is nearly the same on heating and cooling. This is particularly clear in the evolution with 

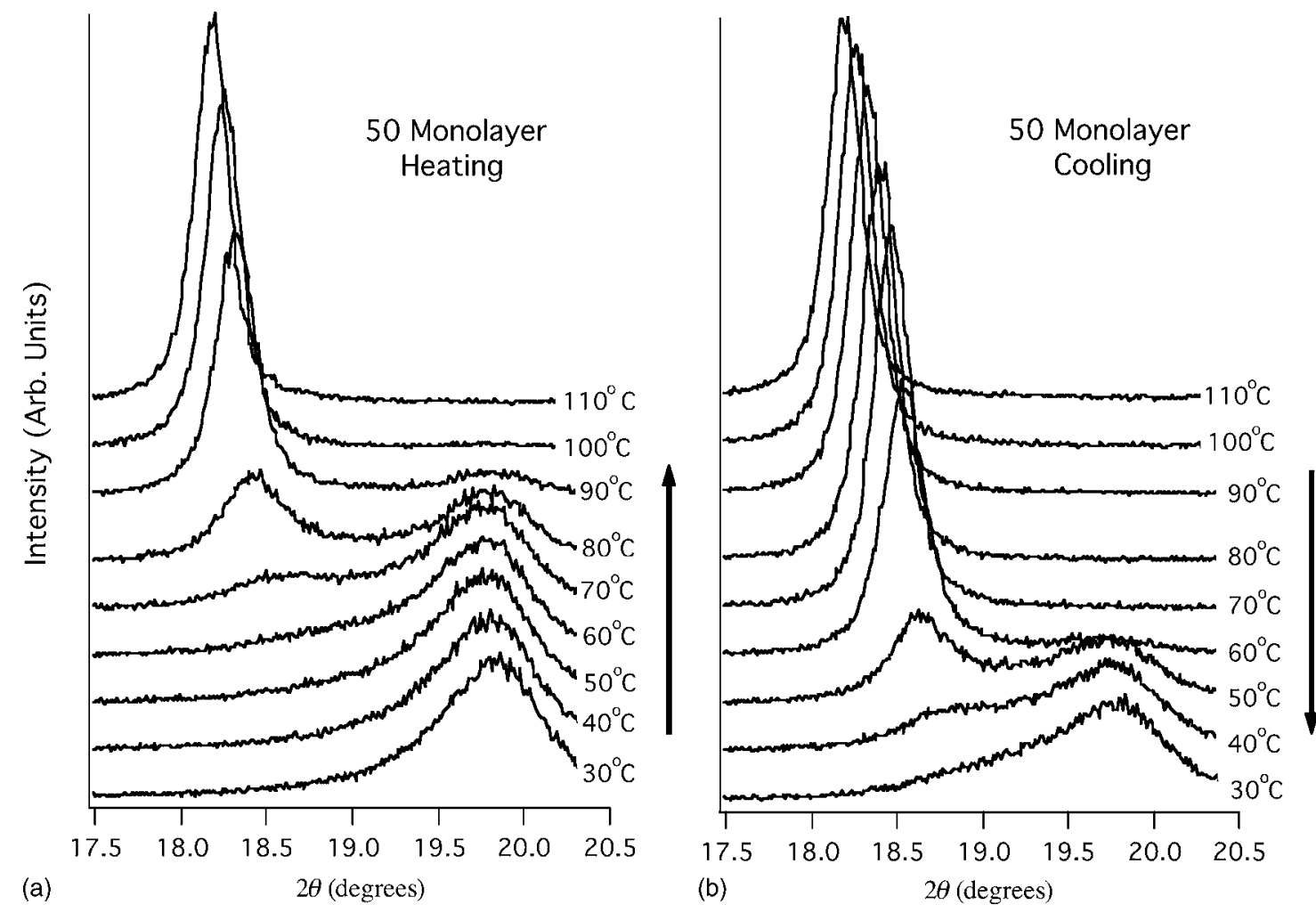

FIG. 3. Temperature dependence of the $\theta-2 \theta$ x-ray diffraction data recorded from a 50-monolayer $\mathrm{P}(\mathrm{VDF}-\mathrm{TrFE})$ sample upon (a) heating and (b) cooling.

temperature of the integrated intensities shown in Fig. 2(c). The integrated intensity of the purely paraelectric phase at $120{ }^{\circ} \mathrm{C}$ is nearly identical in the integrated intensity of the purely ferroelectric phase at $30^{\circ} \mathrm{C}$, supporting the assumption that the structure factors of the two phases are the same. The vertical dashed lines spanning Figs. 2(a)-2(c) mark the approximate lower $\left(65^{\circ} \mathrm{C}\right)$ and upper $\left(100^{\circ} \mathrm{C}\right)$ limits of the zero-field phase coexistence region, showing excellent agreement between the capacitance and x-ray diffraction data. The observed thermal hysteresis and the region of ferroelectricparaelectric phase coexistence observed in the interlayer spacing near the phase transition is consistent with previous studies of bulk and LB films of $\mathrm{P}(\mathrm{VDF}-\mathrm{TrFE})$ as well as with ultrathin Langmuir-Blodgett films of P(VDF-TrFE) ${ }^{10,27}$ For example, Legrand's excellent XRD studies of thick solventcast films of $70 / 30$ copolymer ${ }^{10}$ determined a coexistence region of $85-110{ }^{\circ} \mathrm{C}$ upon heating and $68-58{ }^{\circ} \mathrm{C}$ upon cooling, a little narrower than the results shown in Fig. 3. The increased range of phase coexistence for the LB films may be related to strains due to differential thermal expansion between the film and substrate.

As noted, the relative proportion of each phase is equal to the integrated intensity of its (110) peak, making it easy to follow the conversion from one phase to the other. Figure 2(c) shows that as the temperature of the sample was increased, the amount of material in the ferroelectric phase gradually decreased, while the proportion of the paraelectric phase increased. Conversely, upon cooling, the relative proportion of material in the paraelectric phase remained approximately constant until about $65^{\circ} \mathrm{C}$, where the ferroelectric phase began to grow in proportion at the expense of the paraelectric phase. A small amount of the paraelectric phase persisted even at the low temperature limit of $30^{\circ} \mathrm{C}$, indicating that the film was still not in equilibrium possibly due to residual strain, as noted above. This residual paraelectric phase vanished after several days at room temperature.

\section{B. Phase conversion due to an external electric field}

The temperature-dependent $\mathrm{x}$-ray diffraction and capacitance measurements described in the preceding section confirmed the existence of thermal hysteresis in the ferroelectricparaelectric phase transition and established the coexistence of these phases in Langmuir-Blodgett films of P(VDF-TrFE) $70 / 30$, all in the absence of an electric field. We further expect to observe both phase hysteresis and phase coexistence as a function of applied electric field even at constant temperature. ${ }^{19,22,28,29}$ Once again, capacitance and x-ray diffraction measurements were used to probe the effects of applied electric fields on the ferroelectric-paraelectric phase transition in ultrathin Langmuir-Blodgett films of P(VDFTrFE) $70 / 30$.

Previous dielectric studies established that an external electric field raises the ferroelectric-paraelectric phase transition temperature in some materials, the VDF copolymers included. ${ }^{17,19,28,30}$ To demonstrate this effect in the LB copolymer films, temperature-dependent capacitance measurements were made at a series of applied voltages. Figure 4(a) shows the results of such measurements made on a 30monolayer film of $\mathrm{P}(\mathrm{VDF}-\mathrm{TrFE}) 70 / 30$. At zero applied voltage, the result is similar to the data in Fig. 2(a), where the dielectric peaks upon heating and cooling were $(107 \pm 5)$ and $(75 \pm 5){ }^{\circ} \mathrm{C}$, respectively. [The uncertainties quoted for these peak temperatures is mainly the systematic error in the tem- 

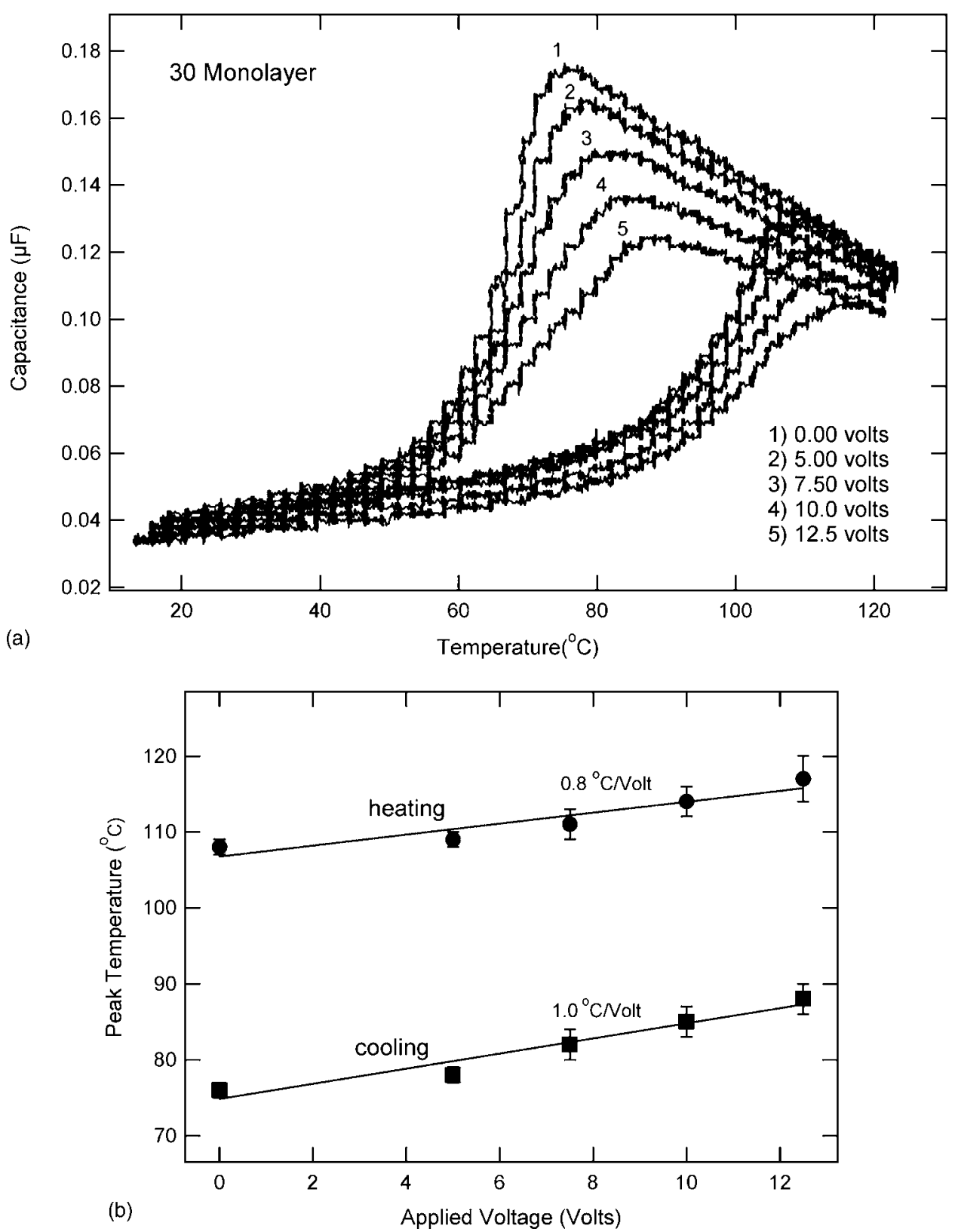

FIG. 4. (a) Temperature-dependent capacitance data recorded from a 30monolayer P(VDF-TrFE 70:30) sample at five different values of applied voltage. (b) The temperature of the peak capacitance for heating $(\mathbf{O})$ and cooling ( $\mathbf{\square})$ plotted as a function of applied voltage. perature measurement, which will be the same for all measurements. The statistical errors associated with locating the peak capacitance are represented by the error bars in Fig. 4(b).] The zero-field dielectric peak temperatures for the 30monolayer film are a little lower than those of the 50monolayer film, perhaps due to the slight thickness dependence of the phase transition temperature. ${ }^{21,31}$ When a voltage was applied to the sample, the dielectric peak temperature on either heating or cooling was shifted upward, as is usual with a first-order phase transition. ${ }^{19,28,29}$ The increase in peak temperature is approximately proportional to the voltage, as shown in Fig. 4(b). The slopes of the best-fit lines to the peak temperatures for heating and cooling are nearly identical and are given by $0.8 \pm 0.5$ and $1.0 \pm 0.5 \mathrm{~K} / \mathrm{V}$, respectively. With a sample thickness of $53.4 \pm 2.1 \mathrm{~nm}(30 \mathrm{ML}$ at $1.78 \pm 0.07 \mathrm{~nm}$ per nominal monolayer $\left.{ }^{32}\right)$, the rate of increase of the transition temperature with field $\left(\partial T_{C} / \partial E\right)$ is $(0.4 \pm 0.2) \times 10^{-7}$ and $(0.5 \pm 0.2) \times 10^{-7} \mathrm{~K} \mathrm{~m} / \mathrm{V}$ for heating and cooling, respectively. The theoretical value for the shift can be calculated from the Clausius-Clapeyron relation ${ }^{29}$

$$
\left(\frac{\partial T_{C}}{\partial E}\right)_{p}=\frac{1}{\alpha_{0}} \sqrt{\frac{16 \gamma}{3|\beta|}},
$$

where $\alpha_{0}, \beta$, and $\gamma$ are the coefficients of the LandauGinzburg-Devonshire free energy. ${ }^{29}$ For P(VDF-TrFE) $70 / 30$, the values of these coefficients $\operatorname{are}^{33} \alpha_{0}=(7.5 \pm 1.5)$ $\times 10^{7} \mathrm{~J} \mathrm{~m} / \mathrm{C}^{2} \mathrm{~K}, \quad \beta=(1.9 \pm 0.2) \times 10^{12} \mathrm{~J} \mathrm{~m}^{5} / \mathrm{C}^{4}, \quad$ and $\gamma$ $=(1.9 \pm 0.2) \times 10^{14} \mathrm{~J} \mathrm{~m}^{9} / \mathrm{C}^{6}$. To calculate the expected value of $\left(\partial T_{C} / \partial E\right)$ from Eq. (1), we must also take into account the projection of the electric $\mathbf{E}$ field, which is applied along the (110) crystal direction, on the spontaneous polarization $\mathbf{P}$, which is directed along the (010) direction, reducing the effective field strength by $13 \%$. The expected value is then $\left(\partial T_{C} / \partial E\right)_{p}=(2.7 \pm 0.6) \times 10^{-7} \mathrm{~K} \mathrm{~m} / \mathrm{V}$, somewhat larger than the measured values. Table I lists the values of $\partial T_{C} / \partial E$ from 
TABLE I. Comparison of the values for the rate of increase of the transition temperature with electric field obtained from the Clausius-Clapeyron relation and from several experimental measurements in $\mathrm{P}(\mathrm{VDF}-\mathrm{TrFE}) \mathrm{70/30}$ films made by LB deposition and solvent spinning.

\begin{tabular}{|c|c|}
\hline Source & $d T_{C} / d E(\mathrm{~K} \mathrm{~m} / \mathrm{V})$ \\
\hline Clausius-Clapeyron [Eq. (1)] & $(2.7 \pm 0.6) \times 10^{-7}$ \\
\hline Solvent-spun films ${ }^{\mathrm{a}}$ & $2.0 \times 10^{-7}$ \\
\hline Double hysteresis with LB films ${ }^{\text {b }}$ & $(0.7 \pm 0.2) \times 10^{-7}$ \\
\hline $\begin{array}{l}\text { Integrated } x \text {-ray peak intensities, increasing } \\
\text { field (paraelectric to ferroelectric) }{ }^{c}\end{array}$ & $(2.4 \pm 0.2) \times 10^{-7}$ \\
\hline $\begin{array}{l}\text { Integrated } x \text {-ray peak intensities, decreasing } \\
\text { field (ferroelectric to paraelectric) }{ }^{c}\end{array}$ & $(1.3 \pm 0.4) \times 10^{-7}$ \\
\hline Dielectric peaks, cooling $^{\mathrm{d}}$ & $(0.5 \pm 0.2) \times 10^{-7}$ \\
\hline Dielectric peaks, heating ${ }^{\mathrm{d}}$ & $(0.4 \pm 0.2) \times 10^{-7}$ \\
\hline
\end{tabular}

${ }^{\mathrm{a}}$ From Ref. 18 for P(VDF-TrFE) 73/27.

${ }^{\mathrm{b}}$ From Ref. 19, where the LB film thickness was calculated assuming $0.50 \mathrm{~nm}$ per monolayer for the film studied in that work. Those films may have been somewhat thicker than that, which would mean that $d T_{C} / d E$ is somewhat larger than given here.

${ }^{\mathrm{c}}$ From the present work (Fig. 6) assuming a thickness of $1.78 \pm 0.07 \mathrm{~nm}$ per monolayer (Ref. 32).

${ }^{\mathrm{d}}$ From the present work (Fig. 4) assuming a thickness of $1.78 \pm 0.07 \mathrm{~nm}$ per monolayer (Ref. 32).

the current and previous results ${ }^{19}$ along with the predicted theoretical value.

While it has been assumed that we are working in the zero pressure regime, in the most general case, one should consider the effect that both the electric field $E$ and the electrostatic pressure have on $T_{C}$. The shift in $T_{C}$ from both contributions is given by

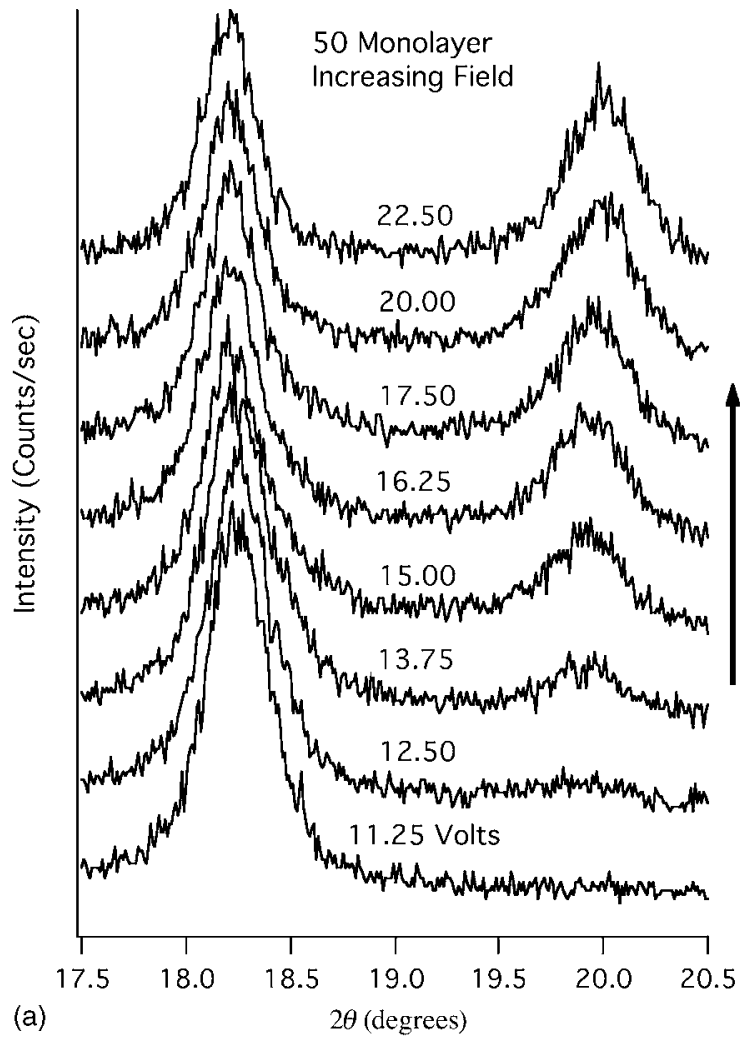

$$
\Delta T_{C}=\left(\frac{\partial T_{C}}{\partial p}\right)_{E} p+\left(\frac{\partial T_{C}}{\partial E}\right)_{p} E
$$

In a thin film capacitor, the electrostatic pressure (Maxwell stress) is quadratic in the electric field by $p=\varepsilon \varepsilon_{0} E^{2}$, where $\varepsilon_{0}$ is the permittivity of free space, $\varepsilon=10$ is the dielectric constant, $^{32}$ and the pressure coefficient is $\left(\partial T_{C} / \partial p\right)_{E}$ $=0.38 \mathrm{~K} / \mathrm{MPa}$ for the $\mathrm{P}(\mathrm{VDF}-\mathrm{TrFE}) 70 / 30$ copolymer. ${ }^{15}$ At the highest applied field $(234 \mathrm{MV} / \mathrm{m}$, or $204 \mathrm{MV} / \mathrm{m}$ projected along $\mathbf{P}$ ), the electrostatic pressure would be $p$ $=3.7 \mathrm{MPa}$, so the first term in Eq. (2) would be $1.4 \mathrm{~K}$, a relatively small contribution to the measured shift of $12 \pm 6 \mathrm{~K}$. [Equation (2) also neglects the tensor aspects connected with the (110) film orientation.] There is a slight indication of a quadratic term in the data shown in Fig. 5, but this is within the experimental uncertainties and so cannot be considered evidence for a contribution from Maxwell stress, the first term in Eq. (2).

Past studies of PVDF in bulk form have demonstrated the conversion from the nonpolar trans-gauche conformation to a polar form. At intermediate fields, Davis et al. were able to convert the nonpolar trans-gauche phase (they assumed this was the bond-ordered orthorhombic $\alpha$ phase, which could be considered antiferroelectric because the individual chains are polar but packed into opposing sublattices) to a polar (i.e., orthorhombic $\alpha_{p}$ ) structure. ${ }^{30}$ Upon application of even higher electric fields, they were able to further convert some of the sample to the all-trans $\beta$ phase, thus implying that the proper sequence of phases was $\alpha-\alpha_{p}-\beta$, which made sense considering the structures of these phases. ${ }^{30}$ However,

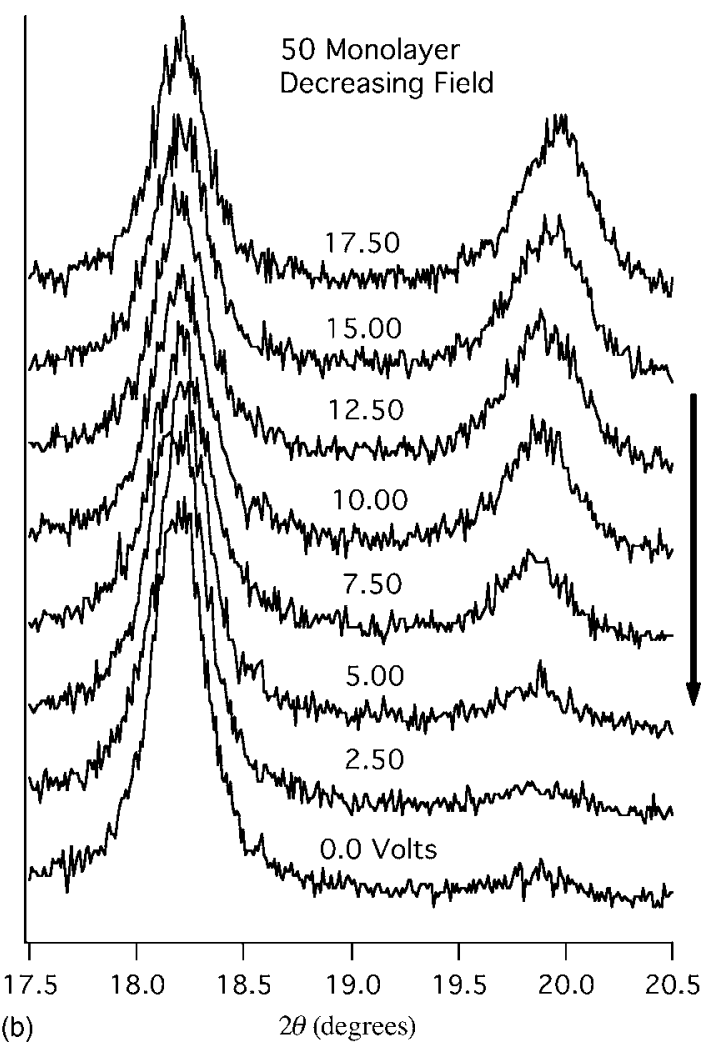

FIG. 5. Electric-field dependence of the $\theta-2 \theta$ x-ray diffraction data recorded from a 50-monolayer $\mathrm{P}(\mathrm{VDF}-\mathrm{TrFE})$ sample at $90{ }^{\circ} \mathrm{C}$ upon (a) increasing and (b) decreasing the electronic field. 


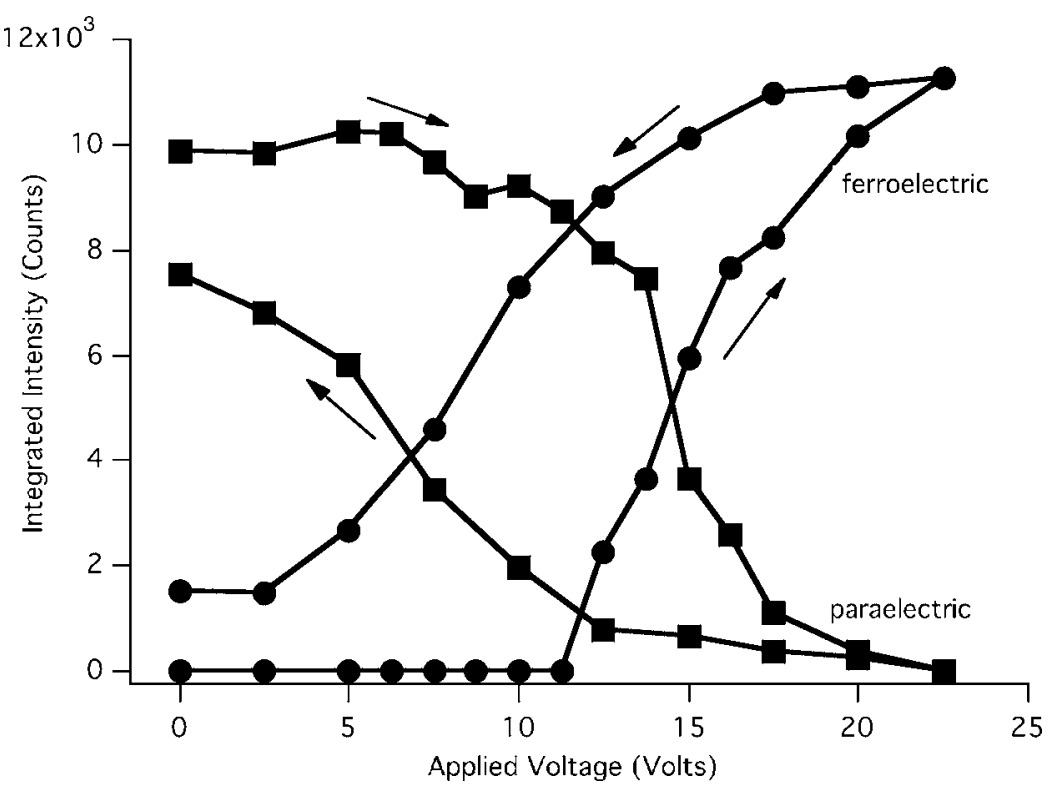

FIG. 6. Integrated intensities of the XRD peaks in Fig. 5 , where the squares ( $\square$ ) indicate the paraelectric phase and the circles $(\bullet)$ indicate the ferroelectric phase. it is important to note that this process was not reversible, so it is not clear that this was the proper sequence of equilibrium phases. Further, there are qualitative differences between PVDF and its copolymers. PVDF normally has a phase transition temperature above the crystal melting point, making direct observation of the ferroelectric-paraelectric phase transition possible only after application of high pressure. ${ }^{14}$ More recent studies have identified the paraelectric phase in VDF copolymers as a hexagonal packing of random trans-gauche chains, ${ }^{12}$ so that the transition sequence is from paraelectric disordered trans-gauche to the ferroelectric ordered all-trans $\beta$ structure. Even in this picture, it is possible for an intermediate phase such as $\alpha_{p}$ to appear upon application of an electric field, but there are no reported observations of such an intermediate phase.

To investigate the field-induced phase transition in the LB copolymer films, we monitored the (110) spacing as a voltage was applied to the sample. A 50-ML sample was first prepared in the paraelectric phase by annealing at $(120 \pm 5)^{\circ} \mathrm{C}$ at zero voltage for $1 \mathrm{~h}$ and cooling slowly to $(90 \pm 5){ }^{\circ} \mathrm{C}$. As expected from the temperature-dependent measurements (Figs. 2 and 3), at $90{ }^{\circ} \mathrm{C}$ and zero bias, the samples exhibit only the paraelectric phase (110) peak at $2 \theta=18.25^{\circ}$ [Fig. 5(a)]. While the temperature of the sample was maintained at $90{ }^{\circ} \mathrm{C}$, a series of XRD measurements (Fig. 5) was recorded with a bias voltage applied in increments first increasing from zero to $22.50 \mathrm{~V}$ and then decreasing back to zero. There was no significant change in the XRD data and no sign of the ferroelectric $\beta$ phase up to a bias of $11.25 \mathrm{~V}(126 \mathrm{MV} / \mathrm{m})$. The $\beta$ phase was first evident at $12.50 \mathrm{~V}$ bias where a weak peak at approximately $2 \theta$ $=19.75^{\circ}(d=4.5 \AA)$ was evident. As the applied voltage was further increased, the ferroelectric peak grew steadily at the expense of the paraelectric peak and appeared to saturate by $20.00 \mathrm{~V}(225 \mathrm{MV} / \mathrm{m})$; there was no additional phase conversion at $22.5 \mathrm{~V}$. Conversely, upon decreasing the applied voltage, there is no significant change in the XRD data until the bias was reduced to approximately $15.00 \mathrm{~V}$, at which point the paraelectric peak intensity began to increase at the expense of the ferroelectric peak. This conversion continued as the applied voltage across the sample was reduced again to zero, where approximately $15 \%$ of the sample remains in the ferroelectric phase.

Application and removal of the electric field converted the sample from paraelectric to paraelectric and back without changing the overall crystallinity. By calculating the integrated intensities of each of the two peaks at each voltage, ${ }^{34}$ we found that the total diffracted signal was constant; the electric field served only to convert between the phases, not to further anneal the sample. This is in contrast to poling studies of PVDF, which did not exhibit a return of the paraelectric phase upon removal of the applied electric field. ${ }^{30}$ Because the cross-sectional area of the x-ray beam was larger than that of the top electrode, not all of the sample was subjected to the applied electric field. We determined the appropriate proportion of this field-free by noting that the phase conversion appeared saturated by $22.5 \mathrm{~V}$. We assumed that the remaining intensity in the paraelectric peak at $22.5 \mathrm{~V}$ came from the part of the film not covered by the top electrode and subtracted this value from intensities at all other voltages to determine the contribution from the portion of the film subjected to the applied electric field.

The net XRD intensity (as determined after subtraction as described in the previous paragraph) plotted in Fig. 6 shows clearly the phase conversion and hysteresis due to the applied electric field. The region of the ferroelectricparaelectric phase coexistence upon increasing voltage is observed to extend from 12.50 to $20.00 \mathrm{~V}$, which for the $89 \pm 4 \mathrm{~nm}$ film thickness corresponds to the range of electric fields from 140 to $224 \mathrm{MV} / \mathrm{m}$. While decreasing the applied voltage, the region of coexistence extends from $15.00 \mathrm{~V}$ $(167 \mathrm{MV} / \mathrm{m})$, marked by an approximate $10 \%$ decrease in the ferroelectric intensity and a corresponding $10 \%$ increase in the paraelectric intensity, down to zero bias voltage, revealing the same metastable ferroelectric phase at $90{ }^{\circ} \mathrm{C}$ as was observed during the heating studies [Figs. 2 and 3]. This is consistent with the fact [see Fig. 2(c)] that a sample polarized at low temperature still retains some ferroelectric phase 
at $90{ }^{\circ} \mathrm{C}$ as it is heated in zero field. Similar results recorded at higher temperature have shown that upon decreasing the field, the lower bound of the coexistence region in fact occurs at a nonzero applied voltage. This indicates the presence of sufficient thermal energy to convert the ferroelectric alltrans chains to paraelectric trans-gauche chains during the time of observation. Unfortunately, this experiment was not consistently repeatable at progressively higher temperatures due to the accelerated electrode degradation and shortcircuiting problems, which occurred when trying to apply large enough voltages across the polymer samples for extended periods of time.

Another way to estimate the rate of shift of the transition temperature with temperature $d T_{C} / d E$ is to use the crossover points in the graph of integrated $x$-ray peak intensities versus temperature [Fig. 2(c)] and versus applied voltage (Fig. 6). In the case of increasing field, the crossover from the paraelectric phase to the ferroelectric phase is at $14.45 \pm 0.5 \mathrm{~V}$. The corresponding temperature shift difference is the difference between the measurement temperature $\left[(90 \pm 0.5){ }^{\circ} \mathrm{C}\right]$ and the crossover temperature $\left[(51.2 \pm 2.0){ }^{\circ} \mathrm{C}\right]$ on cooling from the paraelectric to the ferroelectric phase, as shown in Fig. 2(c). Then the rate of change of transition temperature with field is $\Delta T_{C} / \Delta E=\left[(90 \pm 0.5){ }^{\circ} \mathrm{C}-(51.2 \pm 2){ }^{\circ} \mathrm{C}\right] /[(14.45 \pm$ $0.5) \mathrm{V} /(90 \pm 4) \mathrm{nm}]=(2.4 \pm 0.2) \times 10^{-7} \mathrm{~K} \mathrm{~m} / \mathrm{V}$. The crossover from the ferroelectric phase to the paraelectric phase occurs at $6.75 \pm 0.5 \mathrm{~V}$ on decreasing field (Fig. 6) and at $(80.7 \pm 2){ }^{\circ} \mathrm{C}$ on heating at zero field, resulting in a rate of change of transition temperature with field of $\Delta T_{C} / \Delta E$ $=(1.3 \pm 0.4) \times 10^{-7} \mathrm{~K} \mathrm{~m} / \mathrm{V}$. These values, which are recorded in Table I, are lower than the Clausius-Clapeyron values but higher than the values obtained from the dielectric peaks.

\section{CONCLUSIONS}

The x-ray diffraction and capacitance measurements have shown phase coexistence, phase conversion, and thermal hysteresis as a function of both temperature and electric field in the ultrathin crystalline Langmuir-Blodgett films of $\mathrm{P}(\mathrm{VDF}-\mathrm{TrFE})$ 70/30. Temperature-dependent studies have also further established the qualitative similarities in the phase transition in the highly crystalline ultrathin LB films and the thicker polymorphous solvent-spun and solvent-cast films. The ferroelectric-paraelectric phase transition temperature increases with applied field, confirming results obtained previously using dielectric hysteresis. ${ }^{19}$ There remains a significant discrepancy between the measured values of $d T_{c} / d E$ $\left[(0.4-0.5) \times 10^{-7} \mathrm{~K} \mathrm{~m} / \mathrm{V}\right.$, Table I] and the value 3.1 $\times 10^{-7} \mathrm{~K} \mathrm{~m} / \mathrm{V}$ calculated from the Clausius-Clapeyron relation [Eq. (1)]. In addition it has been demonstrated directly through field-dependent $\theta-2 \theta$ XRD measurements, recorded at a fixed temperature, that it is possible to convert the random trans-gauche paraelectric phase to the all-trans ferroelectric phase with an external electric field. Upon removal of the electric field, the ferroelectric system reverts back to the paraelectric phase, though not entirely at $90{ }^{\circ} \mathrm{C}$. To our knowledge this is the first structural verification of this pro- cess in any ferroelectric system. Moreover, this conversion occurs in a continuous and repeatable manner, consistent with a first-order phase transition that can be modeled by the Landau-Ginzburg-Devonshire mean-field theory.

\section{ACKNOWLEDGMENTS}

Work at the University of Nebraska-Lincoln was supported by the National Science Foundation (ECS-0070245) and the Nebraska Research Initiative, and work performed at the Institute of Crystallography was supported by the Russian Foundation for Basic Research (05-02-16871) and INTAS (1000008-8091).

${ }^{1}$ J. Valasek, Phys. Rev. 17, 475 (1921).

${ }^{2}$ A. J. Lovinger, Science 220, 1115 (1983).

${ }^{3}$ H. S. Nalwa, Ferroelectric Polymers (Dekker, New York, 1995), p. 895.

${ }^{4}$ J. I. Scheinbeim, B. A. Newman, B. Z. Mei, and J. W. Lee, Proceedings of the 1992 IEEE International Symposium on Applications of Ferroelectrics, 1992 (unpublished), pp. 248-249.

${ }^{5}$ H. Kawai, Jpn. J. Appl. Phys. 8, 975 (1969).

${ }^{6}$ T. Furukawa, Phase Transitions 18, 143 (1989).

${ }^{7}$ T. T. Wang, J. M. Herbert, and A. M. Glass, The Applications of Ferroelectric Polymers (Blackie, Glasgow/Chapman and Hall, New York, 1988).

${ }^{8}$ A. J. Lovinger, G. T. Davis, T. Furukawa, and M. G. Broadhurst, Macromolecules 15, 323 (1982)

${ }^{9}$ K. Tashiro, in Ferroelectric Polymers, edited by H. S. Nalwa (Dekker, New York, 1995), pp. 63-181.

${ }^{10}$ J. F. Legrand, Ferroelectrics 91, 303 (1989)

${ }^{11}$ H. Ohigashi, K. Omote, and T. Gomyo, Appl. Phys. Lett. 66, 3281 (1995).

${ }^{12}$ E. Bellet-Amalric and J. F. Legrand, Eur. Phys. J. B 3, 225 (1998).

${ }^{13}$ S. Ikeda, T. Yuki, T. Nojiri, H. Suzuki, and T. Koda, in Electroactive Polymers and Rapid Prototyping, edited by Y. Bar-Cohen, Q. M. Zhang, E. Fukada, S. Bauer, D. B. Chrisey, and S. C. Danforth (Materials Research Society, Boston, MA, 2002), Vol. 698, pp. EE3.7.1-6-EE3.7.6.

${ }^{14}$ K. Matsushige, Phase Transitions 18, 247 (1989).

${ }^{15}$ E. Bellet-Amalric, J. F. Legrand, M. Stock-Schweyer, and B. Meurer, Polymer 35, 34 (1994)

${ }^{16}$ T. Yuki, S. Ito, T. Koda, and S. Ikeda, Jpn. J. Appl. Phys., Part 1 37, 5372 (1998).

${ }^{17}$ G. T. Davis, T. Furukawa, A. J. Lovinger, and M. G. Broadhurst, Macromolecules 15, 329 (1982)

${ }^{18}$ S. Ikeda, H. Suzuki, and S. Nagami, Jpn. J. Appl. Phys., Part 1 31, 1112 (1992).

${ }^{19}$ S. Ducharme, A. V. Bune, V. M. Fridkin, L. M. Blinov, S. P. Palto, A. V. Sorokin, and S. Yudin, Phys. Rev. B 57, 25 (1998).

${ }^{20}$ S. Palto, L. Blinov, A. Bune, E. Dubovik, V. Fridkin, N. Petukhova, K. Verkhovskaya, and S. Yudin, Ferroelectr., Lett. Sect. 19, 65 (1995).

${ }^{21}$ A. V. Bune, V. M. Fridkin, S. Ducharme, L. M. Blinov, S. P. Palto, A. V. Sorokin, S. G. Yudin, and A. Zlatkin, Nature (London) 391, 874 (1998).

${ }^{22}$ S. Ducharme, S. P. Palto, and V. M. Fridkin, in Ferroelectric and Dielectric Thin Films, edited by H. S. Nalwa (Academic, San Diego, 2002), Vol. 3 , pp. $545-591$

${ }^{23}$ K. B. Blodgett, J. Am. Chem. Soc. 57, 1007 (1935); M. C. Petty, Langmuir-Blodgett Films: An Introduction (Cambridge University Press, Cambridge, 1996).

${ }^{24}$ I. V. Langmuir and V. J. Schaefer, J. Am. Chem. Soc. 60, 1351 (1938); A. Sorokin, S. Palto, L. Blinov, V. Fridkin, and S. Yudin, Mol. Mater. 6, 61 (1996).

${ }^{25}$ T. Furukawa, Ferroelectrics 57, 63 (1984)

${ }^{26}$ A. Bune, S. Ducharme, V. M. Fridkin, L. Blinov, S. Palto, N. Petukhova, and S. Yudin, Appl. Phys. Lett. 67, 3975 (1995); S. Palto, L. Blinov, A. Bune, E. Dubovik, V. Fridkin, N. Petukhova, K. Verkhovskaya, and S. Yudin, Ferroelectrics 184, 127 (1996).

${ }^{27}$ J. Choi, C. N. Borca, P. A. Dowben, A. Bune, M. Poulsen, S. Pebley, S. Adenwalla, S. Ducharme, L. Robertson, V. M. Fridkin, S. P. Palto, N. N. Petukhova, and S. G. Yudin, Phys. Rev. B 61, 5760 (2000). 
${ }^{28}$ W. J. Merz, Phys. Rev. 91, 513 (1953).

${ }^{29}$ M. E. Lines and A. M. Glass, Principles and Applications of Ferroelectrics and Related Materials (Clarendon, Oxford, 1977).

${ }^{30}$ G. T. Davis, J. E. McKinney, M. G. Broadhurst, and S. C. Roth, J. Appl. Phys. 49, 4998 (1978).

${ }^{31}$ L. M. Blinov, V. M. Fridkin, S. P. Palto, A. V. Bune, P. A. Dowben, and S. Ducharme, Usp. Fiz. Nauk 170, 247 (2000); 43, 243 (2000).
${ }^{32}$ M. Bai, A. V. Sorokin, D. W. Thompson, M. Poulsen, S. Ducharme, C. M. Herzinger, S. Palto, V. M. Fridkin, S. G. Yudin, V. E. Savchenko, and L. K. Gribova, J. Appl. Phys. 95, 3372 (2004).

${ }^{33}$ S. Ducharme, V. M. Fridkin, A. V. Bune, S. P. Palto, L. M. Blinov, N. N. Petukhova, and S. G. Yudin, Phys. Rev. Lett. 84, 175 (2000).

${ }^{34}$ L. S. Zevin and G. Kimmel, Quantitative X-Ray Diffractometry (SpringerVerlag, New York, 1995). 